\title{
Integrating groupware technology into the learning environment
}

\author{
Abdul Naeem Shaikh and Linda Macaulay \\ Department of Computation, UMIST, Sackville Street, Manchester, M60 IQD \\ email:ans2@co.umistac.uk
}

This paper presents the hard lessons learned from the introduction of groupware technology within a final-year software engineering module. The module began in 1997 and is now in its fourth year. The paper provides a detailed account of our successes and failures in each year, and describes what the authors now feel is a successful model for integrating groupware into the learning environment. The paper is important because it provides a longitudinal study of the use of groupware within a learning environment and an insight into the key success factors associated with the use of groupware. Success factors relate not only to the technology but also to social factors such as group facilitation and social protocols, to factors associated with monitoring and assessment, and to factors related to the skills development associated with being a member of a global team.

\section{Introduction}

The challenge of teaching computer-supported co-operative working (CSCW) and groupware concepts to software engineering students using a 'theory and practice' approach was taken by Macaulay, at the Department of Computation, UMIST, United Kingdom. The first author had assisted from the very beginning and, thus, shared the valuable insights and lessons that came in the introduction of groupware and its subsequent use within the learning environment. Although this challenge was met with a degree of success from the very first year (Macaulay, Shaikh and Young, 1998), attempts were made to learn further lessons each year (in terms of what worked and what did not) and to incorporate them into the following year.

The 'CSCW and Software Engineering' module, hereafter called 'CSCW' module, was structured into four main parts followed by a revision and consolidation session. The four parts reflected the groupware's four classifications of time and place (Macaulay et al., 
1998); that is, synchronous-local (meeting using shared drawing tools), synchronousremote (using video-conferencing), asynchronous-local (using co-ordination tools such as Lotus Notes), and asynchronous-remote (using email, bulletin board, and Web technologies). The practical work consisted of a series of software engineering tasks carried out using groupware tools. Each part consisted of lectures, seminars, practical work, assessment and feedback.

The aims of the module were:

1. to expose students to the practical usage of groupware technologies, representative of those which support each of the four situations;

2. to educate students about the technological, organizational and social issues associated with the application of groupware;

3. to encourage students to consider the role of $\mathrm{CSCW}$ (groupware) within software engineering.

The objectives were that, at the end of the module, the student should be able:

1. to compare and contrast groupware technologies;

2. to select appropriate groupware technologies, depending on the social and organizational context;

3. to evaluate their experiences of using groupware technologies;

4. to explain the issues associated with groupware use, in terms of setting and adopting work protocols;

5. to acquire the skills that are required for effective group collaboration and communication;

6. to assess the relevance of groupware support to software engineering activities.

\section{Case studies}

This section describes an in-depth investigation of the use of groupware with the final-year software engineering students for specific software engineering activities. It covers three case studies of the module from 1997 to 1999.

The first sub-section describes the methodology adopted by the authors for the investigation. The second briefly presents the objectives of the different groupware tools being used during the module. The next three sub-sections cover the cases themselves, each describing the context, the evaluation instruments used and the evaluation results.

\section{Methodology}

According to Almstrum (1996):

The introduction of new technologies increases the importance of evaluation in order to untangle the snarl of factors and influences that impinge on how technology is used in 
context . . o our understanding is so rudimentary that even flawed data are more useful than no data at all, especially if the flaws are recognized. Sometimes just the process of evaluation is revealing, whatever the outcome. An unsatisfactory answer can still lead us to a better question.

To carry through this spirit of Almstrum, the authors adopted an action-research approach to understand the issues surrounding the introduction of groupware and its application within a learning environment. Action research is an effective learning process that compares theory and practice. It involves the participation of the investigator in the investigation itself and relates experiences to prior knowledge (Cao, Laribi, Léonard, Parchet and Zellweger, 1996; Skok and Wardley, 1998). The first author's active participation in the case studies included setting and managing the groupware infrastructure, providing the training, and facilitating the students with their use of groupware tools to carry out the assigned software engineering activities.

For each case study, both quantitative and qualitative data were collected to enable a rich understanding of the introduction and application of groupware. Quantitative data sources included the system log of groupware tools, questionnaires to assess the work group characteristics and to measure the awareness of the functionality provided by the groupware tools. Qualitative data sources included ongoing observations of the authors during each session and the study as a whole, the verbal feedback of a number of students after each session, threaded discussions with the students, and the students' final assessment reports of groupware experiences and its potential in software engineering.

\section{Groupware tools used: objectives and use}

\section{BSCW}

BSCW (Basic Support for Co-operative Work) (GMD-FIT, 2000) was used to support information-sharing and to provide a platform for collaboration between the students and the authors, and between the students themselves. The BSCW was used as follows:

- A repository for module documents. For the lecture notes and schedule, for advertising jobs from employers who were recruiting in CSCW, for details of the research papers that the students were expected to read and for class tests.

- A platform for threaded discussion. Students were asked to read a research paper prior to a class and to consider four or five questions set by the lecturer. Then in the class, the students were divided into small groups and each group was given one of the questions to answer. One member of the group made a short presentation on the result of the group discussion. After the class a second member of the group then 'posted' the answer to the question on the shared workspace. So on BSCW the lecturer had set the question, a student then gave an answer, then other students and the lecturer responded to the answer and so on, thus an online threaded discussion took place. In this way the discussion on particular topics continued throughout the week and indeed throughout the module.

- A means of communication through email. The shared workspace was set up specifically for the students and authors on this module. Entry was by password and a 
directory of valid email addresses and passwords was maintained. This restricted access by anyone outside the module but also made it easier for contact within the module.

\section{Lotus Notes}

The availability of a limited number of Lotus Notes (IBM, 2000) licences restricted each student of the module (student numbers varied from 8 to 42 in the three modules) full access to the Lotus Notes system. Therefore, in the first two years, a single session of three hours was arranged in which students carried out threaded discussions to brainstorm and prioritize the distributed group project issues. However, in the third year of the module, to demonstrate the potential of Lotus Notes as a platform for collaborative system development, the students were given a demonstration of a research prototype being developed by a research student using the Lotus Notes platform.

\section{Video-conferencing}

The limitations of video-conferencing facilities, such as multimedia PCs with video camera, also restricted the availability and extended exposure of video-conferencing to the students. However, a number of short-duration video-conferencing sessions were arranged for the students. In the third year of the module, a special demonstration session of desktop and powerful studio-based video-conferencing was organized at the specially built video-conferencing facility of Manchester Computing Centre. The objective was to enable students to compare the experiences with those described in the literature.

\section{GroupSystems}

The GroupSystems (Ventana-corporation, 2000) tool was used in the second and third years of the module. The objective was to enable students to gain a first-hand experience of working in electronically facilitated meetings. The task for the students was to help the academic staff to address and select seven issues most critical to the success of the distributed projects.

Each GroupSystems session started with a brief introduction of the aims of the task and the agenda of the meeting. This was followed by the brainstorming stage in which the students typed in ideas, read them and then discussed them. Later, different categories were created and the ideas were classified accordingly. This was followed by the voting stage in which each student prioritized the list of major issues, that is, categories, according to his/her opinion. At the end of the session, the final meeting minutes were created, printed and shown to the students for quick discussions. A copy of the meeting report was then emailed and uploaded on BSCW for further discussions.

\section{Case Study I: CSCW' 97 module}

The first case study is concerned with the use of a shared Web-based workspace (Macaulay et al., 1998), namely BSCW, and the practical demonstration of other groupware tools (such as Lotus Notes) in the module. The shared workspace was used as a repository for module documents, a platform for threaded discussions and a means of communication through email.

The exposure to video-conferencing and Lotus Notes was limited to short practical sessions, between one and two hours. A video-conferencing session was arranged using CU-SeeMe (White-Pine, 2000) with audio, video, chat and whiteboard. The collaborative 
task was created for groups of three students, which involved brainstorming and evaluating ideas. The brainstorming and evaluation task consisted of each student having a different part of a task case study to read on the current manual accounting system of a small bookshop. The first stage was to share this information with the other group members and represent the system by a simple diagram. The second stage was to brainstorm a list of possible features of an automated system for the bookshop. The third stage was to prioritize this list into three releases, with the most important features going in the first release.

The exposure to Lotus Notes was also limited to a single session of three hours in which a threaded discussion session was carried out. Students were first provided with a short training session in accessing the Lotus Notes discussion database through a Web browser, and then asked to brainstorm and discuss the assigned task. The task was to help the academic staff to address and select seven issues most critical to the success of the distributed projects.

\section{Evaluation techniques}

No emphasis was given to the evaluation of the use of Lotus Notes and video-conferencing because of the limited exposure. However, students were asked to comment on the potential of these tools in software engineering in their final assessment reports. The BSCW was used throughout the module, and we wanted answers to three questions to evaluate its use:

A) Was a shared workspace the right groupware tool to use for this group?

B) Did the students know how to use it?

C) To what extent did they actually use it?

A) To answer the question: 'Was a shared workspace the right tool?', the authors used some of the work of Roberts (1994). He has developed a technique for assessing the characteristics of a group and the properties of the information being processed. The technique takes the form of a questionnaire (see Appendix 1). His work had been extended by Bate and Travell (1994) to allow a thorough evaluation of the work group and processes in order to choose the appropriate groupware technology. They have recommended ideal characteristics, based on their experiences, for different groupware technologies, such as messaging and information-sharing. The actual characteristics of the group are compared with the ideal characteristics for using a particular type of groupware.

The questionnaire is in four parts. The first part considers the attributes of the group itself, generally assessing the 'groupness' in terms of cohesiveness, sharing, location, adaptability and intelligence. The second part assesses the type of activity being carried out by the group in terms of autonomy, variability, interaction and thought. The third part is concerned with the properties of the information being processed in terms of quantity, type, confidentiality, sources and flow. The fourth and final part asks questions related to the acceptance and take-up of the technology and is classed as 'business issues'.

B) To answer the question: 'Did the students know how to use the shared workspace, $B S C W$ ?', we devised a BSCW feature checklist (see Appendix 2). For each feature available on BSCW we asked whether the students were aware of the feature, how many times they used it, and whether they found it easy to use. 
C) To answer the question: 'To what extent did the students actually use BSCW?', we used the log of BSCW usage. Each time a user read a message, replied to a message, created a document, cut or deleted from a document, logged in or off, an entry was made in a transactions log. This was accessed at various points on the module and analysed.

\section{Evaluation results}

A) The fifteen students were asked to complete the questionnaire, and as recommended by Roberts (1994), the average score for each question was plotted onto a 'spider diagram', as shown in Figure 1. The continuous lines are the actual results from the CSCW module whereas the dotted lines are the ideal results. The top spider diagram shows the 'groupness' of the group and the group activities (the first two parts of the questionnaire). The CSCW student group appeared to lack cohesiveness and interaction with each other when compared with the ideal for using a shared workspace. The bottom spider diagram shows the properties of the information being shared and the business issues. Here there is a good match between the ideal and the actual, particularly in terms of the shape of the diagram. The values on the right-hand side of the diagram are lower than the ideal but still follow the same overall shape.

The spider diagrams (Figure 1) suggest that the use of shared workspace was on the whole an appropriate choice for the CSCW module. However, the lack of cohesiveness within the group and the low level of interaction between the students may point to some problems. There are also some indications that the quantity of information the students had to share was insufficient.

B) The feature checklist was completed by the students towards the end of the module. The results indicated that the majority of the students were aware of only 41 per cent of the features offered by BSCW. However, they reported that those features they did use were easy to use.

C) The BSCW log analysis, undertaken part way through the module, indicated that only about half of the students used BSCW regularly. However, when students were shown the analysis by the lecturer, the number of accesses increased considerably.

\section{Case Study 2: CSCW' 98 module}

The second case study is concerned with the second year of the CSCW module. The main philosophy and the groupware infrastructure remained the same as in the previous module. However, a new groupware tool, GroupSystems, was introduced and a task was designed so that students could gain an experience of electronic meetings. The number of students who finally opted for this module was 42 , which showed its popularity. Students were divided into four roughly equal groups. Four tutors were assigned, one to assist each group. The technical support remained the responsibility of the first author with the added responsibilities of managing the technical support, facilitating his group, and at the same time helping the other tutors to understand the context (based on the author's previous year's experience).

\section{Evaluation techniques}

The structure of the module and the use of groupware tools remained the same, and so did the evaluations. The exposure to video-conferencing and Lotus Notes again remained 


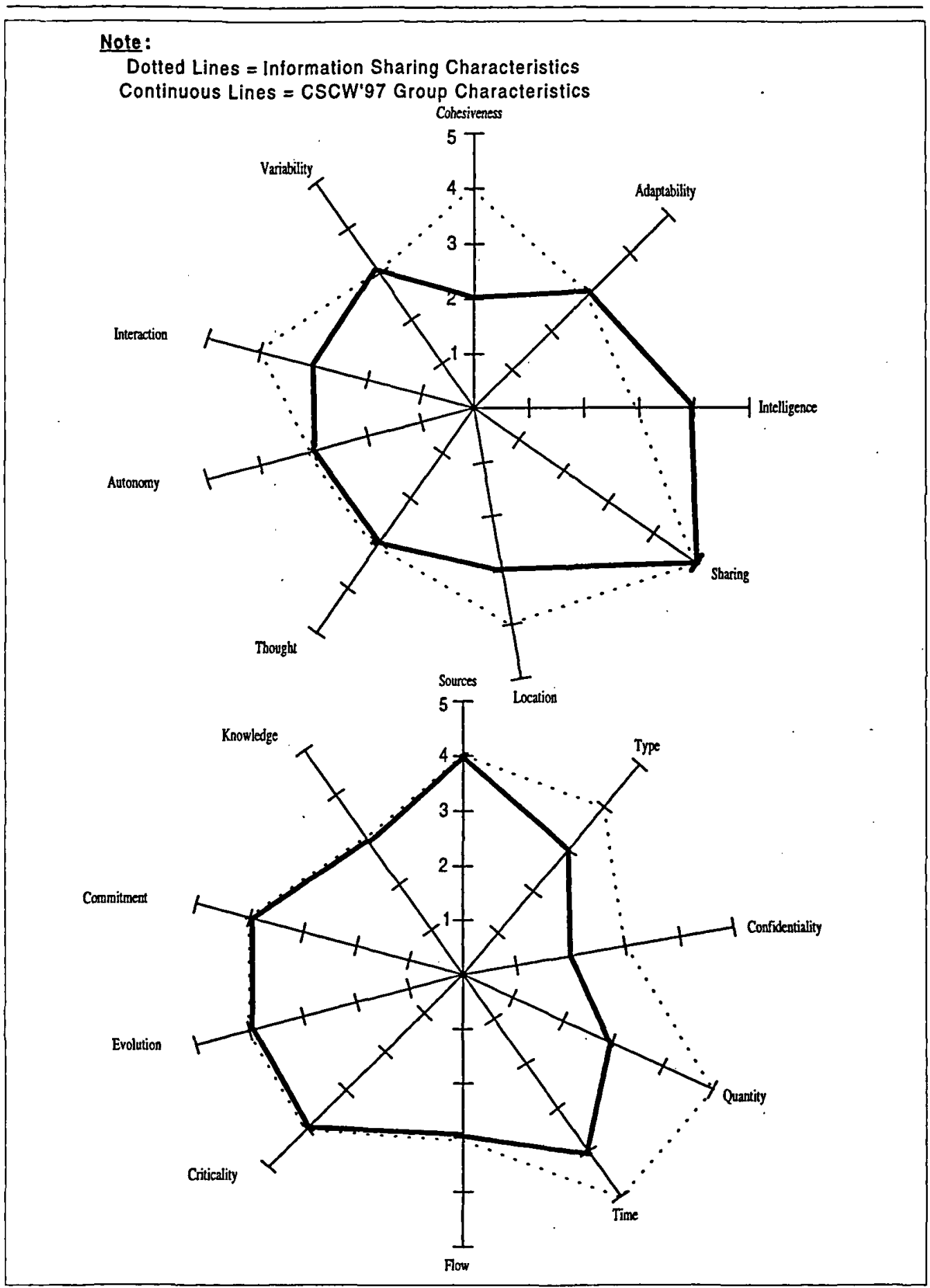

Figure 1: CSCW'97 Group characteristics 'Spider Diogram' 
limited and identical to the previous module, except that a single session of one hour for each group on GroupSystems was arranged, in which the same task of Lotus Notes was repeated.

\section{Evaluation results}

Since there were four groups, each group was separately evaluated by using the spider diagram, BSCW feature check list and the BSCW log analysis. The results of the four spider diagrams were almost the same as that of the previous module. Each group displayed the weaknesses in cohesiveness, interaction, information-sharing, criticality of the task and the quantity of information as compared to the ideal sharing. However, the four spider diagrams follow the same overall shape that indicates BSCW as an appropriate choice for the module.

The results of the BSCW feature checklist indicated that the majority of students were aware of only 38 per cent of the features offered by BSCW. This small drop in the percentage, as compared to the previous module, may be because of the training provided at the start of the module. Some of the students, who attended at the start and received the training, left the module while new students opted in later. Also, it remained impossible to organize another BSCW training session in the middle of the module. Again, students reported that those features they did use were easy to use.

The BSCW log analysis results presented a similar pattern, of an increase in the use of BSCW after the summary was shown in the class which then decreased again. About half of the students used BSCW regularly and explored its other features. Since BSCW does not provide a tool for $\log$ analysis, it was difficult and time-consuming for other tutors to summarize the students' transactions on the BSCW.

\section{Case Study 3: CSCW '99 module}

The final case study is concerned with the third consecutive year of running the CSCW module. The main philosophy and groupware infrastructure remained similar to the previous years. The surprising feature of this case study was the steep drop in the number of students who finally opted for the module, that is, around 40+ students attended in the first week but later only. 8 opted for this module.

The main reason for this change was not that the module had lost its popularity, but there was stiff competition from another module that started in the same term by the same lecturer, named 'Electronic Commerce: Theory and Practice'. Most students had to choose just one, so they decided in favour of the other module.

This term we were expecting a high number of students and as such had made the necessary preparations, for example, starting with the same four experienced tutors and deciding to delay the training of BSCW till the third week. Also, the BSCW server was installed in the department, which helped to address the previous years' problems of slow access and navigation, and which might be one of the reasons for students' low interactions.

A few major changes in the introduction of groupware technologies this year included the following: 
- A dropping of the practical exercises of video-conferencing and Lotus Notes because of the limitations, as mentioned earlier. Also, instead of these tools having a positive impact, students were getting a poor impression of these tools as very unreliable.

- The replacement of the short video-conferencing practical sessions with a visit to a specially built video-conferencing laboratory at the Manchester Computing Centre.

- An extended training and a practical session of GroupSystems.

- A demonstration of the research prototype developed using the Lotus Notes platform by a Ph.D. student.

BSCW, as usual, remained the main groupware tool of the module. All students were put in one group, assisted by one tutor.

\section{Evaluation techniques}

The first and the third evaluation questions ( $A$ and $\mathrm{C}$ ) and the instruments used to answer them remained the same. However, instead of using 'BSCW feature check list' to evaluate the use of BSCW, a discussion thread was initiated on BSCW. The aim was to enable students to record and discuss their immediate experiences (positive or negative) with BSCW.

\section{Evaluation results}

The spider diagram questionnaire was used at two stages: at the mid-term (after week 6) when the students had received the BSCW training and had started using it, and at the end of the module (Week 10). Figure 2 shows the results of the two stages drawn on a spider diagram, along with the ideal characteristics, to examine the improvements achieved between mid and end-term (if any).

The spider diagram shows that at the mid-term students lacked cohesiveness and information sharing. However, the interaction with each other was satisfactory, other factors remaining close to the ideal. The bottom spider diagram, which shows the properties of the information being shared and the business issues, again has a weak match between the ideal and the actual, particularly in terms of the shape of the diagram. The values on the right-hand side of the diagram are much lower than the ideal. In particular the amount of information, and time that students could make available alongside their other commitments, comes very low down.

The end-of-term results (thick lines) show marked improvements in different group characteristics, especially cohesiveness, information-sharing, and amount of information. These results show that the students were able to improve their group characteristics in the second half of the module. However, the importance (criticality) of the task on BSCW is low in both questionnaires, which remains an issue that needs to be addressed. Some students cornmented that there had been too much workload from other modules' assignments.

As in the previous year, the BSCW log analysis was undertaken part-way through the module. The log analysis showed similar results, with about half of the students using BSCW regularly and exploring its other functions. When the log summary was presented in the class, the immediate outcome was a sharp increase in students' access to BSCW in terms of reading and posting a few articles or replying to the questions, but this slowed down over time until the next log summary. 


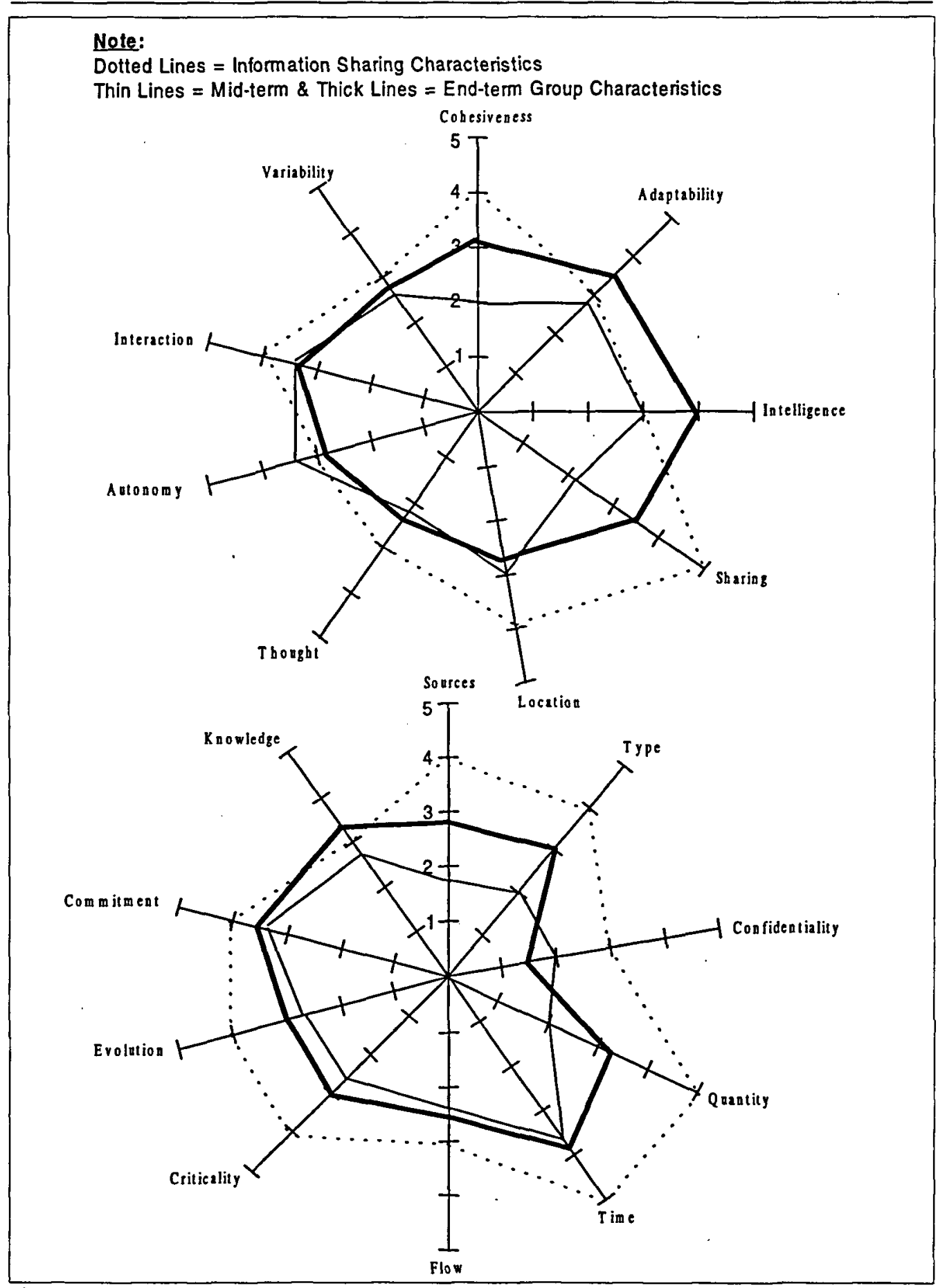

Figure 2: CSCW'99 mid- and end-term group characteristics 


\section{Discussion}

The exposure of students to video-conferencing and Lotus Notes in the modules remained very limited, because of the unavailability of specialized equipment for video-conferencing and a limited number of Lotus Notes licences. Students found difficulty while using audio and video, in part due to their high expectations but also because of not using the equipment properly, and associated technical problems (for example with audio the clipping of words/sentences or total loss of audio, and with video inconsistent, slow refresh which resulted in fragmented pictures). The whiteboard was easy to use, and thus was used cxtensively for the task.

The change in the video-conferencing session in the third year, by a visit to a videoconferencing studio, was welcomed by the students. They reported positively about the experiences gained while observing state-of-the-art demonstrations of remote conferencing. Similarly, in the third year, instead of carrying out thread discussions, the demonstration of a research prototype developed using Lotus Notes platform was reported as having been useful. The experience of attending electronic-facilitated meetings was described as very exciting and instructional. The positive features reported included more participants and less time required, no dominance, anonymous input, support for the thinking process, access to external information, and automated record-keeping. Some problems that students observed included reading an idea or writing while brainstorming, typing mistakes, slight dominance on the basis of typing speed, and the seat configuration (the meeting was arranged in a computer laboratory where the seats were located line by line, rather than round the table).

BSCW was used for the entire length of each module, and was found an appropriate tool to use. In the first two years, the BSCW server at GMD Germany was used, which created uncomfortably long times for access and navigation within the workspace (sometimes even failures). This problem was addressed in the third year when the BSCW server was installed at the local departmental laboratory. However, it created some unexpected problems later, for example, when the BSCW server crashed near the end of the term and could not get immediate attention from support staff because of other high priority engagements. It appeared that some work was needed to make the group more cohesive and communicative with each other prior to its use. Students found the features they tried easy to use, but it appears that they would have benefited from a period of more formal training. (Students were given a quick demonstration of the main features and then left to learn the rest themselves).

The analysis of the usage log is helpful for the lecturer in seeing who is participating in discussions outside formal class time. Once students know that their work is being monitored in this way they tend to increase their amount of usage. However, an increase in quantity does not necessarily represent an increase in quality and may be misleading for the lecturer. Also, monitoring alone could not buy in the students' commitment, especially as they remained very busy with other modules' assignments.

Although the assessment procedures were modified from 100 per cent examination-based to an assessment that included reward for each group's performance, these changes proved to be insufficient to overcome significantly the earlier described weaknesses, such as lack of cohesiveness and information-sharing, and criticality of the task. Some reward for these practical exercises, along with a group's performance, may change the results. 
The issues experienced in the three case studies are presented in the table below, categorized under the headings of technological, organizational and social issues.

Categories
Technological issues
1. Low-cost hardware and software (e.g. PCs, CU-SeeMe)
2. Lack of availability of groupware resources (e.g. Lotus Notes and GroupSystems
licences)
3. Lack of availability of appropriate work environment (e.g. round- table meeting
room facility for EMS, specially built and arranged studio for video-conferencing)
4. Lack of robust and high-bandwidth network infrastructure
5. Unreliability of tools (e.g. audio and video)
6. Over-expectation with the groupware tools
7. Different interfaces for each groupware tool (e.g. BSCW and VC)
8. Lack of assessment of practical work (e.g. use of BSCW)
9. Lack of required commitment and priority given by the support staff while
addressing the technical problems, which consequently affected the students' general
impression of the groupware tools (e.g. 'GroupSystems is unreliable!')
10. Little time for technical and social training
11. No facilitation training for the tutors
12. Students were expected to spend some of their personal time in leaming the
groupware tools
13. The schedule of running the module in the last term of the degree
14.A new way of working, with which the students were not familiar
15. Uneven distribution of work (e.g. some students participated more and/or
contributed better-quality work)
16. Mistrust of the reliability of the technology (early failures and availability of
alternative tools)
17. Higher priorities given by students to other modules' assignments
18. Asynchronous, which requires work during free convenient time, but most
students could not find the time.
19.Varying commitments of different members

Table I: Summary of the issues related to groupware's introduction and application

\section{Lessons learned}

The aim of these case studies was to gain insights into the introduction of groupware and its subsequent application. These insights and understandings are important, since they are based on the authors' personal experiences of facing real problems and then addressing them within existing constraints. The summary below highlights the main lessons drawn from these case studies and the supporting evidence from the evaluation results (Table 1).

\section{Technology}

- There must be equal availability of all the reliable groupware tools among group members [Issues: 1, 2, 5].

- There should be a robust and high-bandwidth technical infrastructure beneath the groupware and the appropriate environment [Issues: $3,4,16]$. 
- Groupware is an 'off-the-shelf' technology and therefore must be configured to meet the needs of the group [Issues: 6, 12].

- Students, lecturers and (importantly) support staff need training (technical and social) in the effective use of groupware technology [Issues: 6, 9-11, 12, 14, 18].

\section{Culture}

- There should be a cohesive reward system based on the group's performance and their level of practical participation [Issues: 8, 15, 17, 19].

- There should be a collaborative culture that would make the group members more cohesive and motivated [Issues: 15, 19].

- The technical support staff and tutors need to accept a different relationship with students, working together to support students and participating in the learning [Issues: 9, 16].

\section{Economics}

- There must be an investment in the technical and physical infrastructure, e.g. robust network, state-of-the-art multimedia PCs, special purpose work set-up, and availability of licences. The worst system will limit the entire group's performance [Issues: 1-4].

- Financial support from the department needs to be justified, maybe through the demonstration of success achieved in the module or by demonstrating improvements in sturlents' skills.

\section{Politics}

- Support for the use of the new technologies is needed at the highest level within a university department [Issues: 9, 13].

- Students need to be motivated to use the technology, for example, by making its use part of the assessed work [Issues: $8,15,17,19]$.

\section{Groupware facilitator}

- A groupware facilitator is needed who can set up the groupware environment, organize and manage its use through effective training and ongoing support [Issues: 7, 9, 14].

- To provide explicit and regular feedback to students [Issues: 14, 16].

\section{Groupware success framework}

Based on the lessons drawn from the above case studies along with the literature review, a framework was constructed as shown in Figure 3. It is called the 'Groupware Success Framework' because it provides a holistic picture of different success elements and their relationships, in order to achieve success with groupware.

The groupware success framework has three main layers; the groupware foundation, the facilitation process, and the groupware success. Below is a brief description of each layer.

\section{Groupware foundation layer}

The groupware foundation layer embodies the four success elements (or factors) for groupware as suggested by Coleman (1997). These elements can be used to address the technological, organizational and social issues related to the introduction and application of groupware. However, inclusion of these elements will not result in success by 


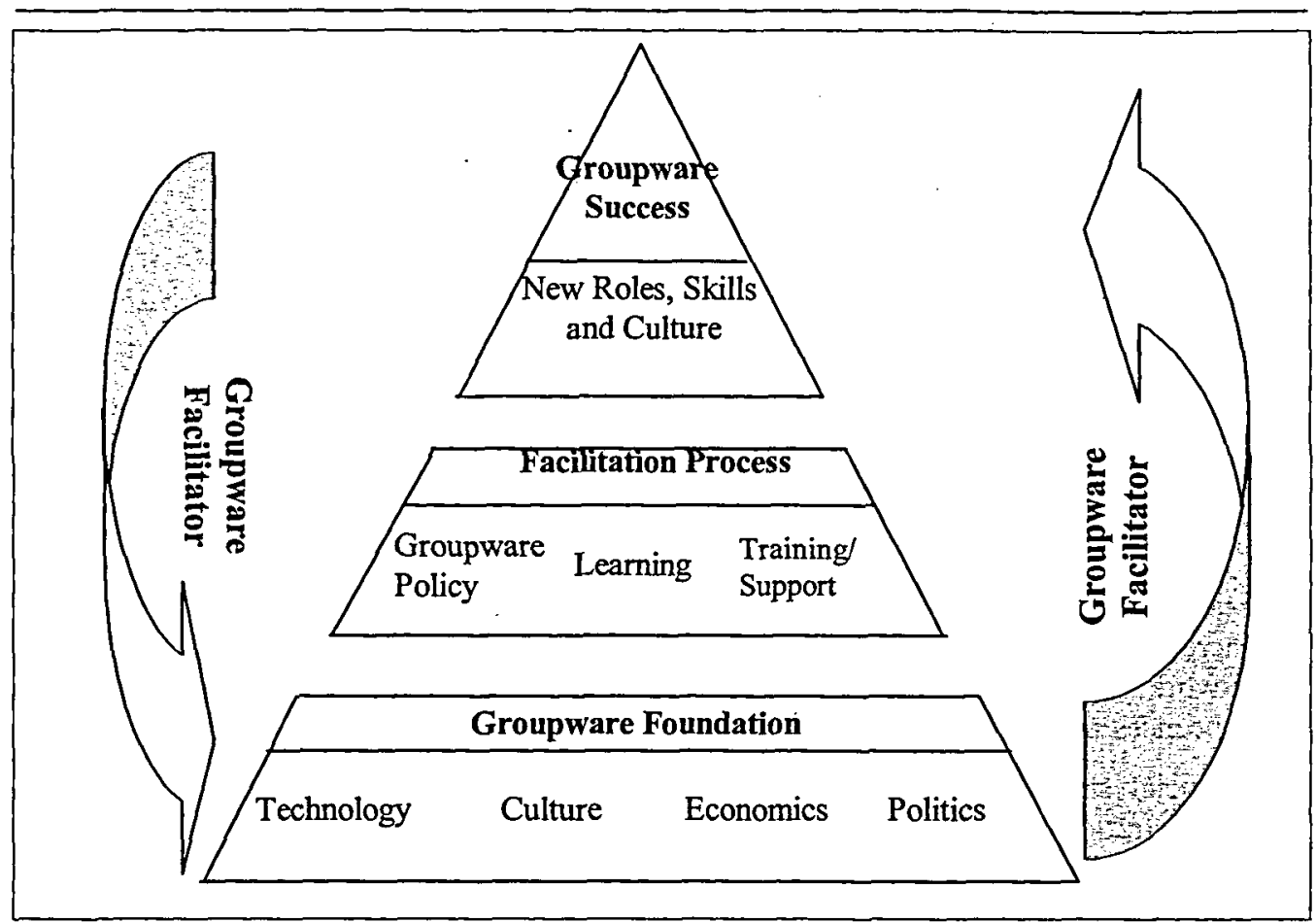

Figure 3: Groupware success framework

themselves; they need a process to manage them to create a collaborative environment. Hence the need for the next layer, the facilitation process.

\section{Facilitation process layer}

This layer is called the 'facilitation process', because it manages the foundation elements and creates the collaborative environment under the supervision of the groupware facilitator. The groupware facilitator is needed because of his/her ability to address and negotiate technological, organizational and social issues simultaneously. Below is a brief description of each facilitation process element.

- Groupware policy. The groupware policy embodies a reward structure based on group incentives and measurements, and a collaborative work environment based on the trust and willingness of the group to share.

- Training and support. There should be task-specific technical training in the groupware system and social support in terms of helping to devise work protocols.

- Learning. Since groupware brings a new way of working, it is important for the group members to know the potential benefits (and risks) of these tools to their daily tasks. Each group is different and so are the work contexts, so groups need to learn what works for them and to keep modifying their work protocols accordingly.

\section{Groupware success layer}

This final layer is dependent on the successful implementation of the two lower layers. It represents the successful introduction and application of groupware, in terms of new roles 
for managers and users, new skills required for efficient use, and the required collaborative culture of trust and sharing.

\section{Conclusion}

This paper has presented the lessons learned from the introduction and application of groupware technology within a final-year software engineering module. The paper has described the detailed accounts of our successes and failures over three years. Finally, it has described what the authors now feel is a successful model for the integration of groupware into the learning environment.

As described in the previous three case studies of the CSCW module, each year the majority of the students agreed that this module had been enjoyable and instructional, but there was a lack of effective collaboration. Therefore, in the fourth year of the CSCW module, a number of changes were made in the design of the course to accommodate more group work and a significant part of the assessment marks ( 25 per cent) were allocated for individual performances in the practical sessions and online discussions along with the group performance. The fourth year of the module is currently being evaluated and the success, or otherwise, of the framework will be the subject of future publications.

\section{References}

Almstrum, V. L., Dale, N., Berglund A., Granger, M., Little, J. C., Miller, D. M., Petre, M., Schragger, P. and Springsteel, F. (1996), 'Evaluation: turning technology from toy to tool: report of the working group on evaluation', ITiCSE '96, Proceedings of the Conference on Integrating Technology into Computer Science Education, ACM Press, 201-17.

Bate, J. S. J. and Travell, N. (1994), Groupware, Alfred Waller Limited.

Cao, N. V., Laribi, A., Léonard, M., Parchet, O. and Zellweger, C. (1996), 'Integrating $\mathrm{CSCW}$ in a cooperative learning environment to teach information systems', Proceedings of the Conference on Integrating Technology into Computer Science Education, ACM Press, 125-29.

Coleman, D. (1997), 'Groupware - the changing environment', in D. Coleman (ed.), Groupware: Collaborative Strategies for Corporate LANs and Intranets, Prentice Hall, 1-38.

GMD-FIT (October, 2000), BSCW Home Page, German National Research Center for Information Technology, http://bscw.gmd.de.

IBM (October, 2000), Lotus Development Corporation, http://www.lotus.com.

Macaulay, L., Shaikh, A. N. and Young, R. (1998), 'Groupware and software engineering: criteria for success', in R. Hazemi, S. Hailes and S. Wilbur (eds.), The Digital University: Reinventing the Academy, London: Springer-Verlag, 245-65.

Roberts, M. (1994), 'Creating the human/technology partnership', in J. S. J. Bate and N. Travell (eds.), Groupware, Alfred Waller Limited, 111-32.

Skok, W. and Wardley, R. (1998), 'A partnership approach in undergraduate business cducation', Proceedings of the 1998 Conference on Computer Personnel Research, ACM Press, 136-44. 
Ventana-corporation (October, 2000), GroupSystems.com, http://www. ventana.com.

White-Pine (October, 2000), CU-SeeMe Networks, http://www.cuseeme.com.

\section{Appendix I: Questionnaire 'To assess work group characteristics' (Roberts, 1994)}

Attributes of the work group:

Was the team operating as a cohesive unit? (Cohesiveness)

Acting as individuals [ (1) (2) (3) (4) (5) ] Cohesive Unit

How adaptable were the team members to change? (Adaptability)

Did not welcome change [ (1) (2) (3) (4) (5)] Welcome change

How intelligent or IT literate were members of the team? (Intelligence)

No IT awareness [ (1) (2) (3) (4) (5) ] IT awareness

To what extent did the team members share their information? (Sharing)

No information sharing [ (1) (2) (3) (4) (5)] Total information sharing

Were the team members located locally or dispersed across diverse locations? (Location) Located in the same office [ (1) (2) (3) (4) (5) ] Spread over diverse locations

Analysis of work group activities:

Did the process carried out by the work group require much thought? (Thought)

Easy, repetitive tasks [ (1) (2) (3) (4) (5) ] Complex tasks

How much autonomy did the team members have? (Autonomy)

Conformed to prescriptive rules [ (1) (2) (3) (4) (5) ] Adapt working environment

To what extent were the team members required to interact with their colleagues?

(Interaction)

Can work in isolation [ (1) (2) (3) (4) (5) ] Must interact to complete task

Did work group activities vary from day to day or were they stable? (Variability)

Same daily activities [ (1) (2) (3) (4) (5) ] Unpredictable daily tasks

Properties of the information being processed:

Did the information arrive from a number of sources? (Sources)

Sole source [ (1) (2) (3) (4) (5)] Wide range of sources

Was the information of a variety of types? (Types)

Same type [ (1) (2) (3) (4) (5) ] Complex range of data types

Was the information being handled of a private or confidential nature? (Confidentiality)

Public information [ (1) (2) (3) (4) (5) ] Strictly confidential information

Were vast quantities of information processed by the work group? (Quantity)

Small amount [ (1) (2) (3) (4) (5) ] Large amount

Was the information held for long periods of time? (Time)

Held for hours [ (1) (2) (3) (4) (5) ] Held for months/ years

Is there a recognized path for the flow of information amongst the work group? (Flow) Rigid information flows [ (1) (2) (3) (4) (5) ] Information takes varying paths 
Business issues:

Was the process being carried out critical to business success? (Criticality) Internal, non-critical process [ (1) (2) (3) (4) (5) ] Critical to business success

Did the activities of the work group evolve? (Evolution) Static processes [ (1) (2) (3) (4) (5) ] Continually evolving area

Was management committed to the introduction/development of work group computing? (Commitment)

Reluctant to change [ (1) (2) (3) (4) (5) ] Strongly committed to change

How knowledgeable was the work force to the benefits of BSCW? (Knowledge)

No knowledge of concepts [ (1) (2) (3) (4) (5) ] Fully understand the benefits

\section{Appendix 2: BSCW feature checklist}

\begin{tabular}{|c|c|c|c|c|c|c|c|c|c|}
\hline \multirow{2}{*}{ FEATURE } & \multicolumn{2}{|c|}{$\begin{array}{l}\text { Were you aware } \\
\text { of this feature? }\end{array}$} & \multicolumn{2}{|c|}{ Did you use it? } & \multicolumn{3}{|c|}{$\begin{array}{l}\text { How many times } \\
\text { have you used it? }\end{array}$} & \multicolumn{2}{|c|}{$\begin{array}{l}\text { Did you find } \\
\text { it easy to use? }\end{array}$} \\
\hline & Yes & No & Yes & No & $\begin{array}{l}\text { Less } \\
\text { than } 5\end{array}$ & $\begin{array}{l}5 \text { to } \\
10\end{array}$ & $\begin{array}{l}\text { More } \\
\text { than } 10\end{array}$ & Yes & No \\
\hline \multicolumn{10}{|l|}{ ACCESS } \\
\hline \multicolumn{10}{|l|}{$\overline{A D D}$} \\
\hline \multicolumn{10}{|l|}{ ARTICLE } \\
\hline \multicolumn{10}{|l|}{$\overline{A D D}$} \\
\hline \multicolumn{10}{|l|}{$\overline{\mathrm{DOC}}$} \\
\hline \multicolumn{10}{|l|}{$\overline{A D D}$} \\
\hline \multicolumn{10}{|l|}{ FOLDER } \\
\hline$\ldots$. & & & & & & & & & \\
\hline
\end{tabular}

\section{Appendix 3: A sample of BSCW log analysis (CSCW' 97)}

\begin{tabular}{|c|c|c|c|c|c|c|c|c|c|c|c|c|c|}
\hline \multirow[b]{2}{*}{ Name } & \multirow[b]{2}{*}{$\begin{array}{l}\text { Log } \\
\text { date }\end{array}$} & \multicolumn{3}{|c|}{ Read } & \multicolumn{3}{|c|}{ Reply } & \multicolumn{2}{|c|}{ Created } & \multicolumn{2}{|c|}{ Cut } & \multicolumn{2}{|c|}{ Delete } \\
\hline & & Papers & Comments & $\begin{array}{l}\text { Week- } \\
\text { wise Plan }\end{array}$ & Jobs & Papers & Comments & Papers & Comments & Papers & Comments & Papers & Comments \\
\hline & $16 / 03 / 97$ & 6 & 3 & & 1 & 1 & 2 & 1 & 1 & 1 & & 1 & \\
\hline \multirow{4}{*}{$\begin{array}{r}\text { Joh } \\
-\frac{1}{1} \\
1\end{array}$} & $13 / 04 / 97$ & 10 & 7 & 2 & 2 & 3 & 2 & 3 & 2 & 1 & & & \\
\hline & $17 / 06 / 97$ & 20 & 3 & 1 & 3 & 3 & 4 & 2 & 2 & 2 & 1 & 2 & 1 \\
\hline & Total & & \multicolumn{2}{|c|}{58} & & \multicolumn{2}{|c|}{15} & \multicolumn{2}{|r|}{11} & \multicolumn{2}{|r|}{5} & \multicolumn{2}{|r|}{4} \\
\hline & $16 / 03 / 97$ & 3 & 4 & & & & 1 & & 1 & & 1 & & \\
\hline Wongl & 1 3/04/97 & 6 & 10 & 2 & 2 & 1 & I & 1 & & & & & \\
\hline & $17 / 06 / 97$ & 5 & 2 & 1 & 1 & 2 & 1 & 2 & 1 & 1 & & & \\
\hline & Total & & 3 & & & & 6 & & 5 & & 1 & & 1 \\
\hline
\end{tabular}

